\title{
EchoGéo
}

16 | 2011

Échos de Turquie

\section{Les effets de l'émigration paysanne dans les Andes équatoriennes : une lecture photographique}

\section{Nasser Rebaï}

\section{(2) OpenEdition}

\section{Journals}

Édition électronique

URL : https://journals.openedition.org/echogeo/12382

DOI : 10.4000/echogeo. 12382

ISSN : 1963-1197

\section{Éditeur}

Pôle de recherche pour l'organisation et la diffusion de l'information géographique (CNRS UMR 8586)

Référence électronique

Nasser Rebaï, «Les effets de l'émigration paysanne dans les Andes équatoriennes : une lecture photographique », EchoGéo [En ligne], 16 | 2011, mis en ligne le 04 juillet 2011, consulté le 25 août 2021. URL : http://journals.openedition.org/echogeo/12382 ; DOI : https://doi.org/10.4000/echogeo. 12382

Ce document a été généré automatiquement le 25 août 2021.

EchoGéo est mis à disposition selon les termes de la licence Creative Commons Attribution - Pas d'Utilisation Commerciale - Pas de Modification 4.0 International (CC BY-NC-ND) 


\title{
Les effets de l'émigration paysanne dans les Andes équatoriennes : une lecture photographique
}

\author{
Nasser Rebaï
}

\section{Introduction}

1 Dans la majorité des études sur les migrations internationales, les analyses ne concernent que les espaces d'arrivée. Pour G. Simon, cela constitue d'ailleurs l'une des «limites de l'approche habituelle des faits migratoires" (2008:12), même si ces derniers temps, divers travaux ont posé un regard éclairé sur les espaces d'émigration (Cortes, 2000 ; Faret, 2006).

2 L'objet de cet article est de proposer une vision des transformations en cours dans les Andes équatoriennes, et plus particulièrement dans la province de l'Azuay où depuis plusieurs années, on assiste à une véritable hémorragie démographique. Dans la paroisse ${ }^{1}$ Octavio Cordero Palacios, située en périphérie de Cuenca (population estimée à 400000 habitants), nos enquêtes ont montré que sur un échantillon de 38 familles paysannes, $50 \%$ de la main-d'œuvre avait quitté la localité pour partir travailler aux Etats-Unis au cours des trois dernières décennies. Dans ce contexte, on observe des mutations profondes, tant au niveau de l'usage du sol que dans les rapports sociaux, ce qui n'est pas sans rappeler le point de vue de J-Y. Marchal (1998), pour qui la ruralité relève bien plus d'un dynamisme constant et d'une certaine forme de complexité, que d'un monde figé qui ne ferait que s'opposer à l'urbain. 
Carte 1 - Localisation de la paroisse Octavio Cordero Palacios

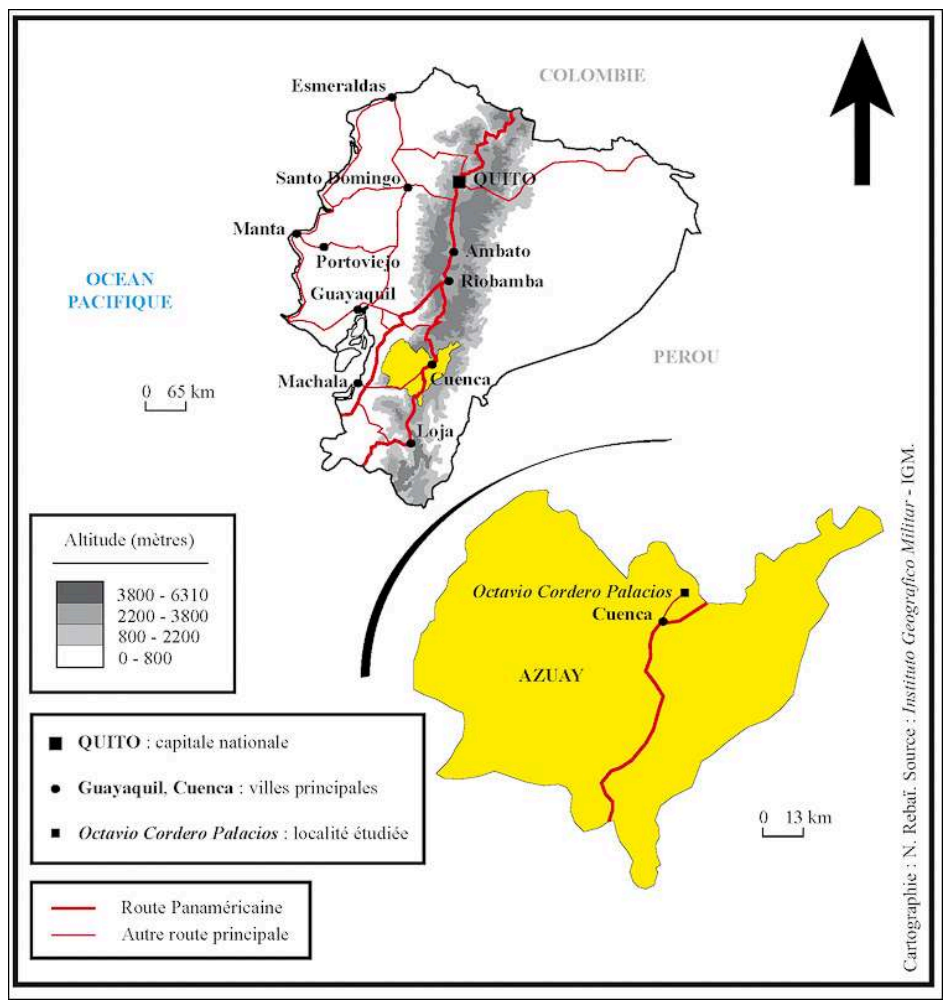

Source : Instituto Geografico militar - IGM ; cartographie : N. Rebaï.

3 Notre ambition est donc de donner un point de vue géographique des effets de l'émigration, en cherchant à nous distinguer des publications équatoriennes les plus récentes, la plupart appartenant aux champs de la sociologie et de l'anthropologie (Ramírez Gallegos et Ramírez, 2005; Herrera et al., 2006). C'est pourquoi nous souhaitons accorder une place privilégiée à l'image, pour traiter de manière plus pertinente des mutations spatiales dans cette région des Andes.

\section{L'image comme outil d'analyse}

Plus qu'une simple illustration, la photographie est pour nous un objet de compréhension des dynamiques paysannes, un support visuel à partir duquel notre réflexion prend forme. Il s'agit précisément de guider le regard de l'observateur vers des formes particulières du paysage, des couleurs ou des éléments plus symboliques, et de les décrypter ensuite. Le commentaire photographique devient alors le cœur de l'analyse, auquel viennent s'ajouter des éléments plus théoriques.

5 A travers cet article, nous proposons donc de faire une restitution synthétique d'un travail de recherche effectué entre mai 2008 et août 2009. En guise de fil conducteur, nous présenterons une série de photographies qui nous mèneront de la campagne vers la ville, et chacune d'entre elles fera l'objet d'une description, d'une interrogation et d'un commentaire précis. Ainsi, nous pourrons montrer comment la migration participe d'une part à la transformation de l'agriculture paysanne et, d'autre part, à la redéfinition des modes d'approvisionnement urbain en produits frais. 
$6 \mathrm{Au}$ cours de notre investigation, près d'un millier de clichés ont été pris, ce qui témoigne d'une volonté forte de faire de l'image une composante majeure de notre analyse géographique. Nous n'en dévoilerons cependant que sept, pour cibler au mieux notre propos.

\section{Du maïs au maraîchage : un espace agraire renouvelé}

Photographie 1 - Paroisse Octavio Cordero Palacios, province de l'Azuay, Equateur

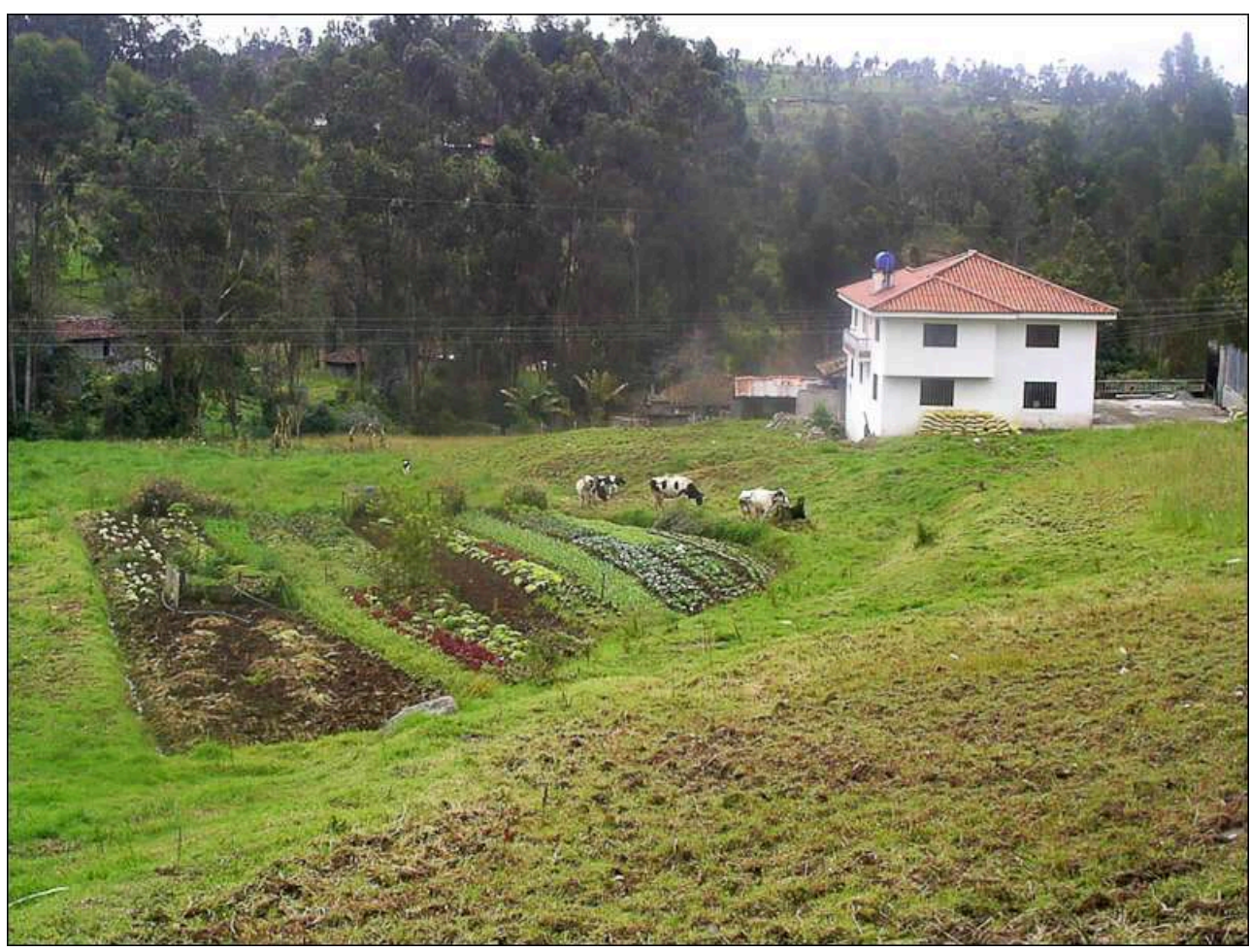

Auteur : N. Rebaï, 2008.

7 Sur la photographie $n^{\circ} 1$, prise de plain-pied pour une vision réduite à une seule parcelle, la présence d'une grande maison construite en dur indique des revenus probablement liés à la migration, dynamique propice au «renouvellement de l'habitat ", comme l'écrivit M. Cote dans son étude des campagnes algériennes (1996). Toutefois, l'apparente modernité de ces nouvelles habitations contraste avec un intérieur plus vétuste, où le mobilier et les équipements restent le plus souvent limités à quelques éléments anciens. La plupart de ces grandes et belles maisons, vues de l'extérieur, ne sont donc que des coquilles vides qui témoignent d'une certaine réussite migratoire chez de nombreuses familles paysannes, sans pour autant bouleverser les habitudes domestiques.

8 Ce qui nous intéresse le plus néanmoins, se trouve tout autour de cette maison. L'espace est majoritairement dédié aux pâturages, au milieu desquels on retrouve un potager d'environ $300 \mathrm{~m}^{2}$. Trois vaches pâturent au piquet à proximité de petites rangées de salades, de choux et de brocolis qui semblent prêts à être récoltés. Quelle est donc l'origine de cet usage du sol? 
Compte tenu du manque de main-d'œuvre, mais aussi des faibles rendements et des maigres bénéfices attendus, les familles paysannes de la paroisse Octavio Cordero Palacios ont progressivement sacrifié les cultures de cycles longs (céréales, tubercules, fève, haricot et petit pois) pour se consacrer davantage à l'élevage laitier, comme en témoigne l'augmentation de $185 \%$ des superficies pâturées dans la localité entre 1991 et $2001^{2}$ (Institut d'Etudes et de Recherches Sectorisées de l'Equateur - IERSE). La vente quotidienne de lait ou de fromages assure aux exploitations des rentrées monétaires régulières, contrairement au maïs, qui même en cas de bonne récolte, ne permet d'obtenir des revenus qu'une fois par an. En outre, la production laitière est un moyen pour les foyers paysans d'assurer leur propre sécurité alimentaire dans un contexte où le manque de main-d'œuvre les contraint à délaisser les cultures vivrières traditionnelles.

10 Toutefois, la paroisse Octavio Cordero Palacios n'est pas un cas isolé. Entre 2000 et 2008, les superficies cultivées dans la province de l'Azuay ont diminué de $65 \%$ (Institut National de Statistiques et de Recensements - INEC), tout cela bien entendu dans un contexte de forte émigration. En plus de l'élevage, le maraîchage s'est aussi beaucoup développé : il correspond à l'utilisation plus rationnelle de la main-d'œuvre disponible, par un travail plus intensif sur des superficies réduites. Ainsi, la production de légumes (salades, choux, oignons, carottes, etc.) couvre en grande partie les besoins alimentaires des foyers ruraux tout en permettant le développement d'une activité commerciale dynamique.

\section{Agriculture commerciale et ravitaillement urbain}

\section{Photographie 2 - Foire hebdomadaire de Miraflores à Cuenca}

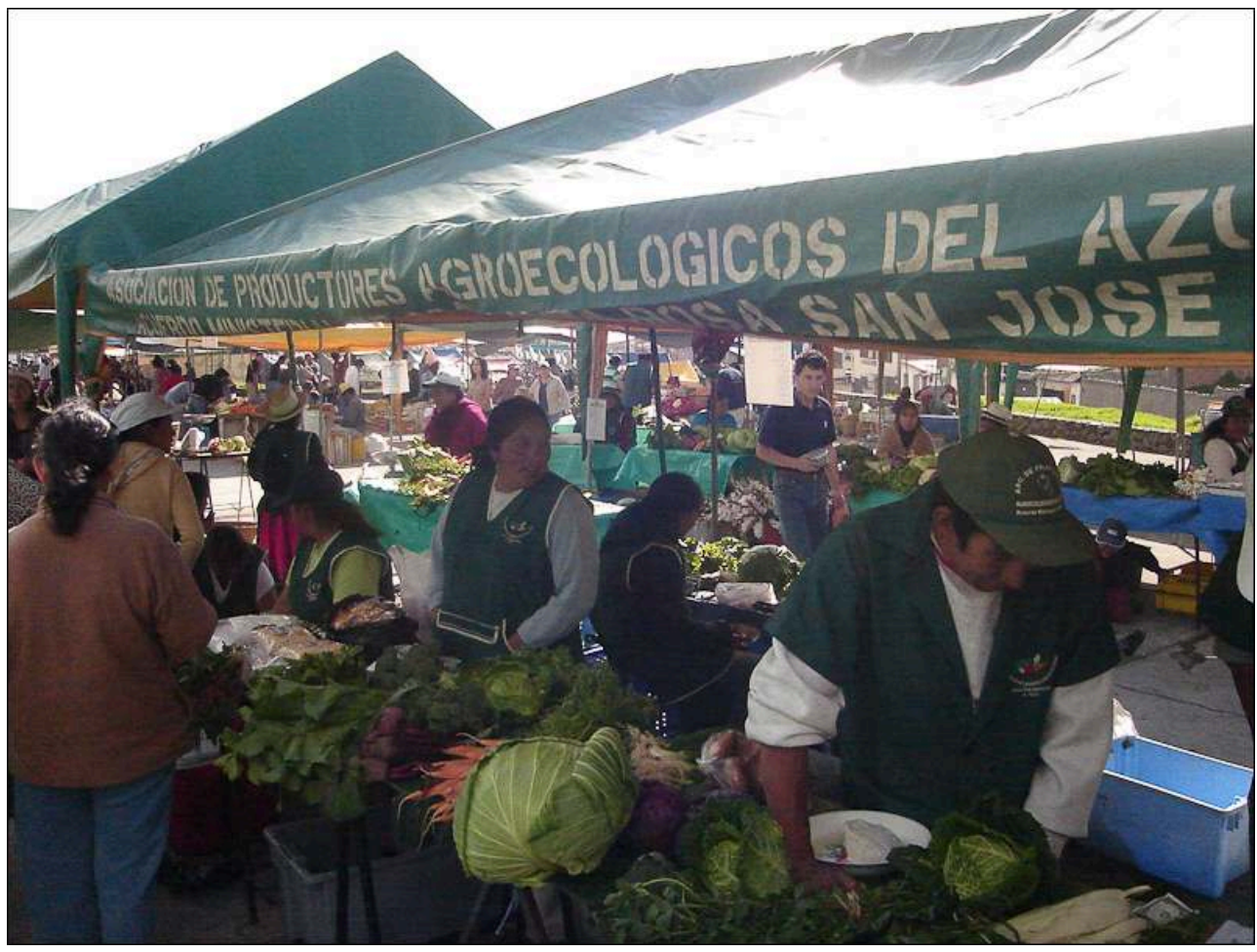

Auteur : N. Rebaï, 2009. 
$11 \mathrm{Au}$ centre de la photographie $\mathrm{n}^{\circ} 2$, une paysanne originaire de la paroisse Octavio Cordero Palacios vend ses produits maraîchers à la foire de Miraflores, l'une des plus importantes de Cuenca. Depuis près de dix ans, elle se rend en ville deux fois par semaine, chargée de paniers contenants légumes et fromages, tandis que son mari travaille aux Etats-Unis. Seule dans son exploitation, l'activité commerciale lui prend du temps, mais elle parvient tout de même à obtenir des revenus réguliers. Comme ses voisins de stand, elle porte un uniforme vert, celui de l'Asociasión de Productores Agroecologicos del Azuay ${ }^{3}$, comme il est indiqué au-dessus d'elle.

12 Sur plusieurs autres marchés cuencanais, on retrouve d'autres vendeurs originaires de la périphérie rurale, habillés des mêmes uniformes, ce qui les distingue de la foule d'intermédiaires partageant avec eux les espaces de vente. Dans quelle mesure alors le contexte migratoire a-t-il favorisé l'émergence d'une agriculture marchande dans la province de l'Azuay?

13 Puisque le manque de main-d'oeuvre a contraint de nombreuses exploitations à s'orienter vers le maraîchage, certains paysans en ont profité pour répondre à la demande urbaine en produits frais. C'est ainsi que depuis plusieurs années on assiste à la croissance du «vivrier marchand» (Chaléard, 1996) dans la province de l'Azuay. Cependant, l'insertion des producteurs locaux dans l'économie urbaine n'aurait pu se faire sans appui institutionnel. Sur ce point, le Programme d'Agriculture Urbaine (PAU) de la Municipalité de Cuenca et le Centre de Reconversion Economique de l'Austro ${ }^{4}$ (CREA) ont joué un rôle capital en créant deux associations de producteurs agroécologiques qui garantissent à près de 300 familles paysannes un accès régulier au marché urbain régional.

14 De l'avis des administrateurs des marchés cuencanais, la présence de producteurs régionaux sur les espaces de vente a redonné du dynamisme à l'activité marchande. Parce qu'ils proposent des produits de qualité à des prix plus bas que ceux pratiqués par les intermédiaires, ceux que l'on nomme les agroécologiques attirent des clients de plus en plus nombreux. Sur certaines foires, on assiste même à une forme de fidélisation entre vendeurs et consommateurs urbains, ce qui n'est pas sans déplaire aux techniciens du PAU et du CREA qui se targuent désormais d'avoir créer un pont entre la ville et sa périphérie rurale.

15 Dans un certain nombre d'exploitations, les revenus agricoles sont aujourd'hui plus importants et plus réguliers, dépassant parfois ceux de la migration. Dans la paroisse Octavio Cordero Palacios, certaines familles gagnent en moyenne 40 dollars par semaine, rien que par la vente de légumes frais. Toutefois, ce sont bien les produits laitiers qui procurent la plus grande part des revenus, les ventes directes sur l'exploitation ou sur les marchés urbains pouvant atteindre 70 dollars par semaine dans certains cas.

16 Si le contexte migratoire semble a priori défavorable à l'agriculture paysanne, il conduit paradoxalement à sa renaissance, du moins en partie. L'influence urbaine joue d'ailleurs un grand rôle pour cela, ce qui n'est pas sans rappeler l'expérience burkinabé décrite par B. Tallet, qui insistait sur « la capacité de réaction du secteur agricole quand la demande solvable existe» (1999: 58). Malgré les contraintes induites par la migration, les agriculteurs familiaux démontrent ainsi qu'ils peuvent agir efficacement pour l'approvisionnement urbain. Néanmoins, cette dynamique ne concerne pas 
l'ensemble des exploitations, entre lesquelles on observe dorénavant des contrastes socioéconomiques flagrants.

\section{Les ventes informelles, une manière de survivre au quotidien}

Photographie 3 - Calle Sangurima dans le centre-ville de Cuenca

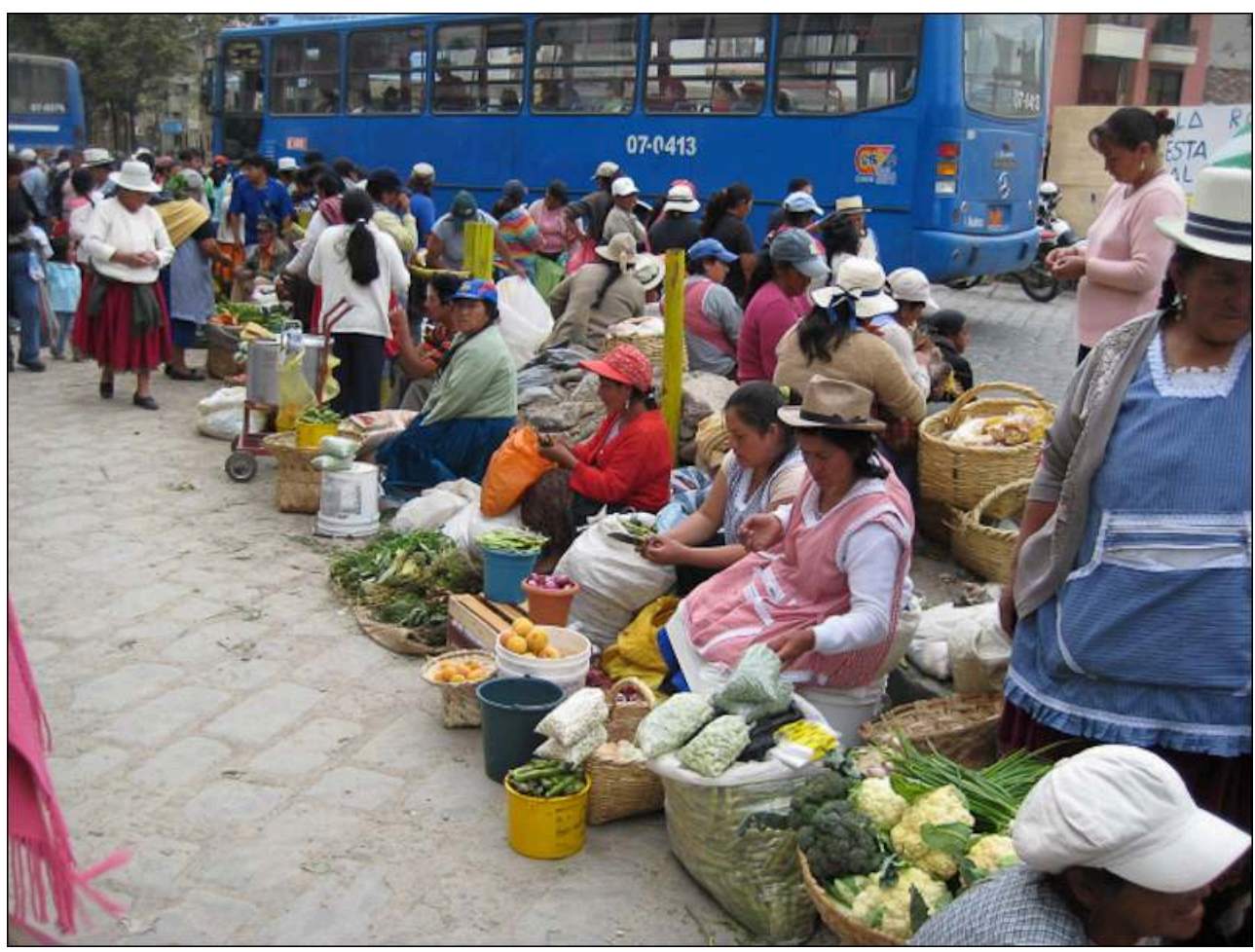

Auteur : N. Rebaï, 2009.

Sur la photographie $n^{\circ} 3$, des vendeuses informelles sont installées à deux pas du marché 9 de Octubre, l'un des espaces de vente les plus populaires de Cuenca. En plein cœur du centre-ville, elles sont nombreuses à vouloir profiter chaque jour de la très grande affluence dans ce quartier où affluent continuellement de nombreuses ménagères. Dans des seaux ou des petits sachets en plastique, elles vendent des fruits à la pièce, des haricots, des fèves, et parfois quelques produits maraîchers. Au milieu de la circulation, entre les bus qui relient le centre ville et la gare routière, elles doivent lutter avec la police qui, de temps à autre, n'hésite pas à les déloger brutalement. Face à cette situation, nous nous interrogeons : quelles différences y a-t-il entre ces vendeuses informelles et les producteurs agroécologiques officiels?

Bien que les objectifs communs du Programme d'Agriculture Urbaine et du Centre de Reconversion Economique de l'Austro soient de réduire la pauvreté paysanne et de proposer une alternative à la migration, dans les faits, les associations qu'ils gèrent n'intègrent que très rarement les populations les plus fragiles de l'espace rural régional, celles qui disposent de très peu de terre et qui ne sont pas en mesure de payer un droit d'entrée élevés. Autrement dit, elles laissent en marge des dynamiques commerciales les familles paysannes qui sont a priori les plus vulnérables et qui auraient un besoin prioritaire d'appuis institutionnels. 


\section{Le jour où le maïs disparaîtra}

Les photographies $n^{\circ} 4$ à 7 nous montrent le maïs dans tous ses états. Du mote (maïs bouilli) qui sert de base à la majorité des plats paysans, de la chicha (boisson légèrement alcoolisée) qui donne du courage aux travailleurs, un tamal (papillote de farine de maïs accompagnée d'un peu de viande et de petits légumes) pour les jours de fête, et les fameux choclos (épis) que l'on mange sur le pouce avec une tranche de fromage frais.

\section{Photographie 4 - Mote}

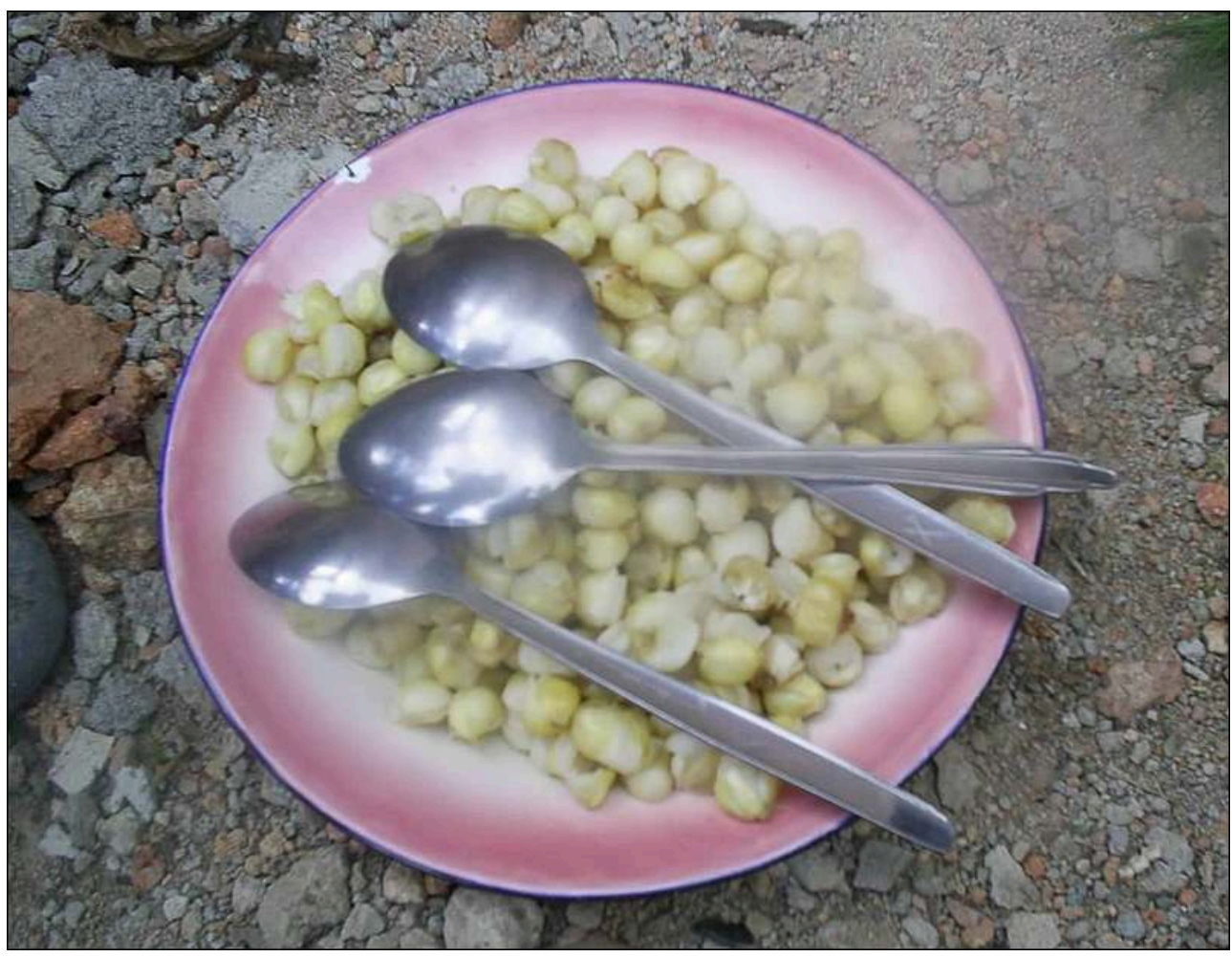

Auteur : N. Rebaï, 2009. 
Photographie 5 - Chicha

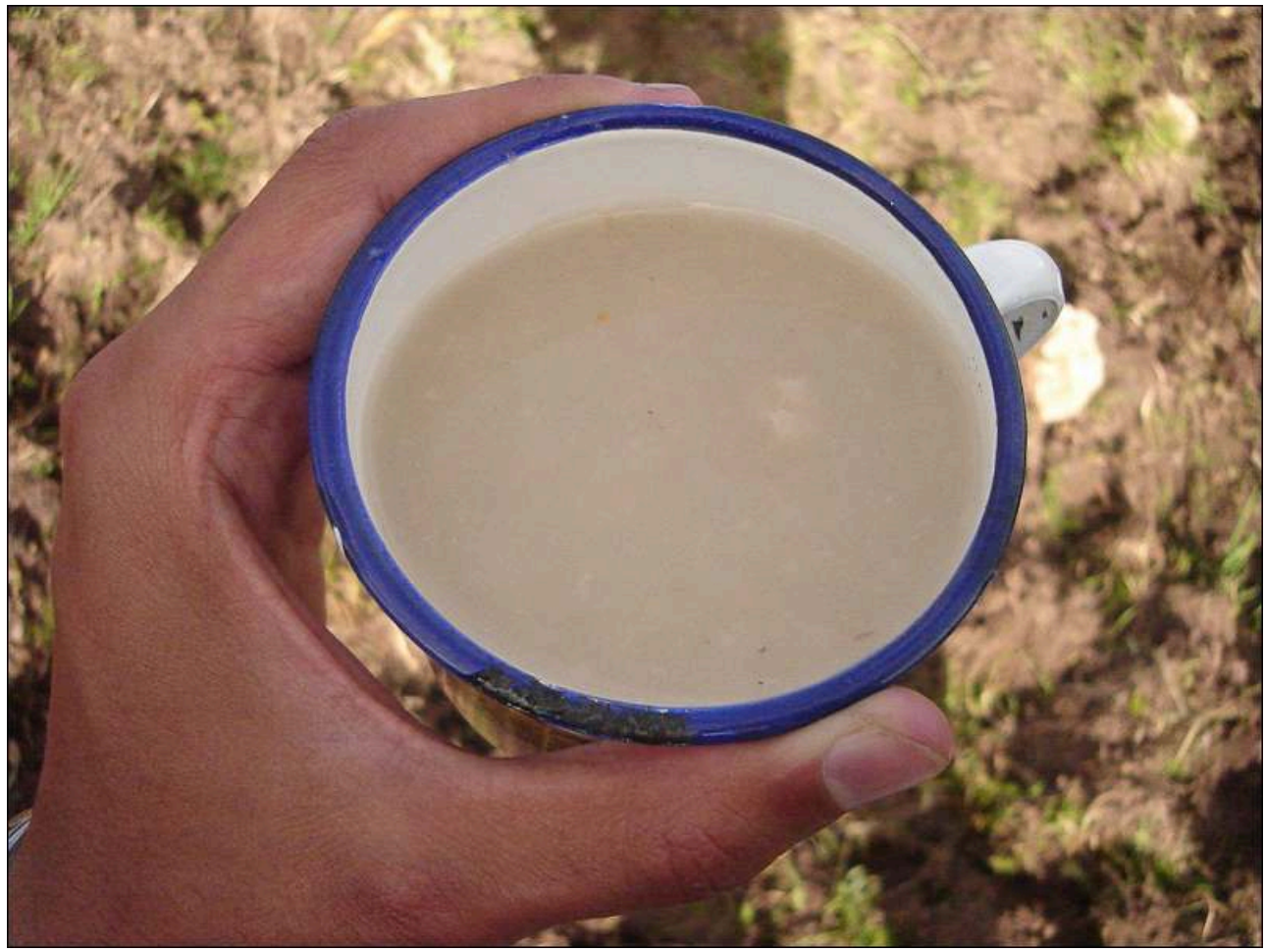

Auteur : N. Rebaï, 2009.

\section{Photographie 6 - Tamal}

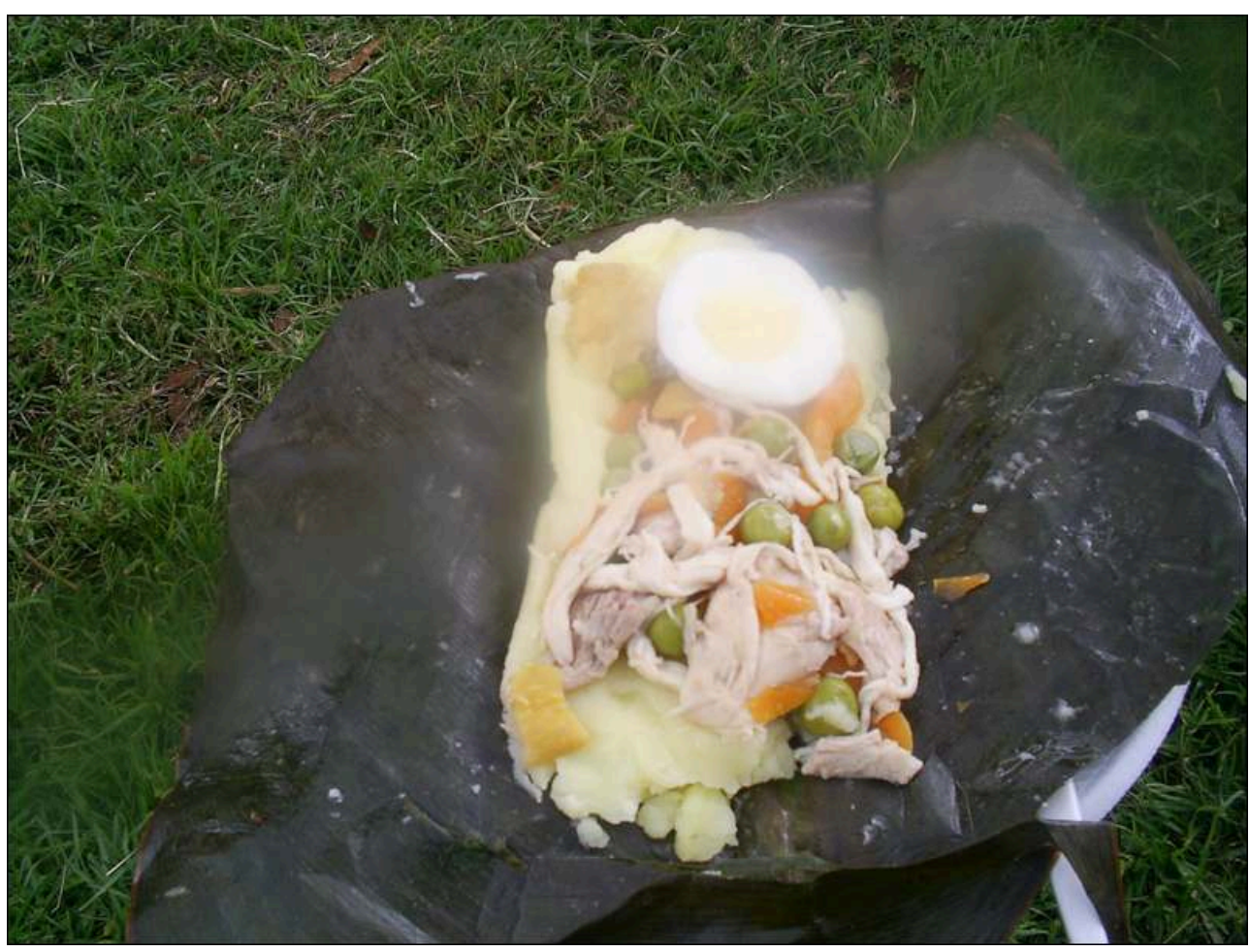

Auteur : N. Rebaï, 2009. 


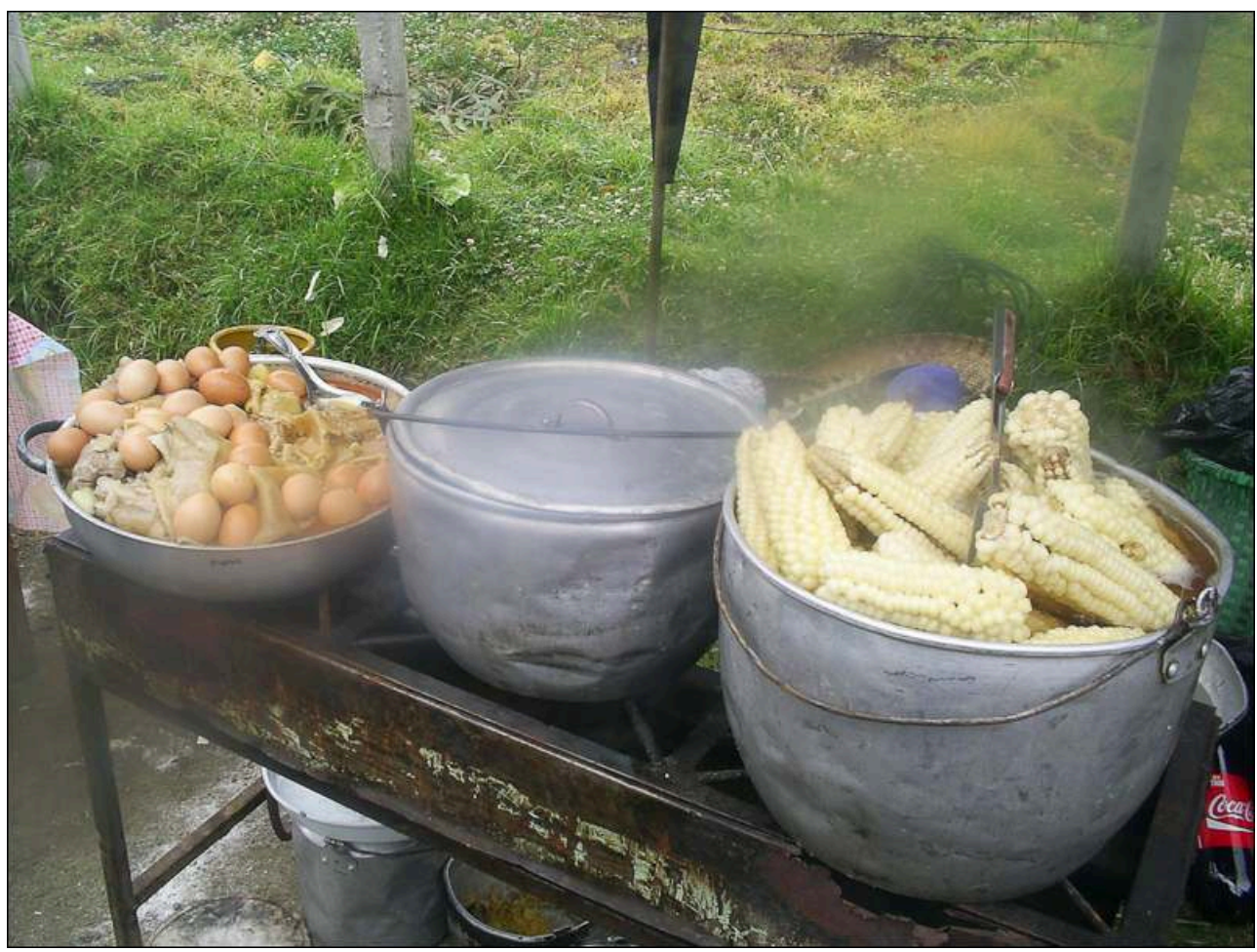

Auteur : N. Rebaï, 2009.

L'avantage de travailler en milieu rural est de pouvoir goûter à la cuisine paysanne. Pour le géographe, il s'agit certainement du meilleur moyen pour devenir « un indigène du territoire qu'il étudie ", comme le dit P. Pélissier dans l'un de ses derniers entretiens (Théodat, 2007: 3). Dans notre cas, une interrogation demeure néanmoins: avec l'émigration, ne verra-t-on pas un jour disparaître la petite paysannerie, et avec elle, l'ensemble de ces plats traditionnels?

21 Si l'on peut envisager à moyen terme des évolutions dans les modes de consommation, en témoignent par exemple la bière et les sodas qui ont progressivement remplacé la chicha ces dernières années, la transformation plus radicale des pratiques agricoles et des systèmes de production, comme c'est déjà le cas, pourrait être problématique.

Dans la province de l'Azuay, le développement du maraîchage et de l'élevage permet à certaines exploitations de se maintenir, mais il est encore trop tôt pour parler de filières qui seront à l'avenir des voies de développement régional. A contrario, la baisse constante des superficies cultivées pourrait être considérée comme un symbole de la « fin des paysans » (Mendras, 1967) dans cette région des Andes. Aussi, la question de la sécurité alimentaire nationale pourrait se poser à l'avenir : les émeutes de la faim qui ont touché nombre de pays du Sud en 2008 ont montré qu'une trop grande dépendance aux importations agricoles, comme c'est le cas en Equateur depuis plus de vingt ans (Peltre-Wurtz, 2004), pouvaient mettre en péril la stabilité d'un État.

23 La priorité est donc pour l'Equateur de mettre fin à l'émigration paysanne, ou du moins de contribuer au maintien des exploitations familiales, et de favoriser la croissance des productions céréalières dans les provinces andines. Pour cela, le retour à une agriculture locale et l'articulation plus évidente entre villes et campagnes pourraient être les premiers pas vers un futur meilleur. Mais nul doute que pour lutter 
efficacement contre l'émigration et son lot de drames affectifs, il conviendrait d'agir pour que les productions subventionnées des pays du Nord ne viennent pas perturber les marchés au Sud. Au-delà même du cas équatorien, ce sont les mécanismes de l'agriculture mondialisée qu'il convient par conséquent de repenser.

\section{Conclusion}

La question migratoire dans les Andes équatoriennes est donc particulièrement complexe. Loin d'être réductible à la seule dimension économique ou sociale, elle suppose en réalité de penser à l'avenir des exploitations familiales. Le processus de "révolution citoyenne ${ }^{6}$ " engagé par le président R. Correa devrait être propice à la promotion de politiques agraires ambitieuses en faveur de l'agriculture paysanne. La nouvelle constitution adoptée en 2008 fait d'ailleurs de la souveraineté alimentaire un "objectif stratégique", pour lequel il importe de "renforcer le développement d'organisations et de réseaux de producteurs ${ }^{7}$ ». Reste à savoir si l'Etat équatorien dispose d'une marge de manœuvre suffisamment importante pour appliquer ces grandes résolutions et mettre fin à la pauvreté paysanne.

\section{BIBLIOGRAPHIE}

Chaléard J-L., 1996. Temps des villes, temps de vivres. L'essor du vivrier marchand en Côte d'Ivoire. Paris, Karthala, $661 \mathrm{p}$.

Cote M., 1996. Pays, paysages, paysans d'Algérie. Paris, CNRS Editions, 282 p.

Cortes G., 2000. Partir pour rester : survie et mutation de sociétés paysannes andines (Bolivie). Paris, IRD Editions, $413 \mathrm{p}$.

IERSE, 2003. Registro de la información cartográfica de la cuenca del Paute. Cuenca.

INEC, 2000. III Censo Agrario Nacional. Quito.

INEC, 2002/2008. Sistema Estadístico Agropecuario Nacional. Quito.

Faret L., 2006. Dynamiques transnationales et développement au Mexique : les enjeux de la migration internationale. Hérodote, $\mathrm{n}^{\circ} 123$, p. 182-198.

Herrera G., Carrillo M-C., Torres A. (eds.), 2006. La migración ecuatoriana. Transnacionalismo, redes e identidades. Quito, FLACSO, $512 \mathrm{p}$.

Marchal J-Y., 1998. La ruralité ou la contradiction. In Gastellu J-M. et Marchal J-Y. (eds.), La ruralité dans les pays du Sud à la fin du XXème siècle, Paris, Karthala, p. 13-25.

Mendras H., 1967. La fin des paysans, innovations et changements dans l'agriculture française. Paris, SEDEIS, $364 \mathrm{p}$.

Peltre-Wurtz J., 2004. Alimentation et pauvreté en Equateur. Paris, IRD/Karthala, 184 p. 
Ramírez Gallegos F., Ramírez J-P., 2005. La estampida migratoria ecuatoriana. Quito, Abya Yala, 239 p.

Simon G., 2008. La planète migratoire dans la mondialisation. Paris, Armand Colin, 255 p.

Tallet B., 1999. Le maraîchage à Bobo-Dioulasso (Burkina Faso) : un dynamisme agricole sous influence urbaine ». In Chaléard J-L. et Dubresson A. (eds.), Villes et campagnes dans les pays du sud: une géographie des relations, Paris, Karthala, p. 47-59.

Théodat J-M., 2007. Entretien avec Paul Pélissier. EchoGéo [En ligne], nº 1. URL : http:// echogeo.revues.org/1660

\section{NOTES}

1. Dans l'espace rural équatorien, la paroisse est la plus petite unité administrative.

2. Il s'agit là des statistiques disponibles les plus récentes.

3. Association de Producteurs Agroécologiques de l'Azuay.

4. L'Austro, correspond au trois provinces australes de l'Equateur, à savoir le Cañar, l'Azuay et le Morona Santiago.

5. Dans l'une comme d'ans l'autre association, chaque nouveau membre doit payer cinquante dollars avant de bénéficier d'un étal pour vendre ses produits.

6. "Révolución ciudadana ».

7. «Fortalecer el desarrollo de organizaciones y redes de productores » (Titre IV, Chapitre 3, Article 281, Alinéa 10).

\section{RÉSUMÉS}

Dans les Andes équatoriennes, les effets de l'émigration paysanne sont partout visibles. Si dans la province de l'Azuay, on assiste à la diminution des superficies cultivées et à la réorganisation des tâches agricoles, on observe par ailleurs l'émergence d'une agriculture commerciale orientée vers le marché urbain régional. En procédant à la description de photographies prises à la campagne comme à la ville, nous chercherons à expliquer l'ensemble de ces mutations, avant de nous interroger plus largement sur l'avenir des exploitations familiales dans cette région.

En los Andes ecuatorianos, los efectos de la migración campesina son visibles por todas partes. Si en la provincia del Azuay, se asiste a la disminución de las superficies cultivadas y a la reorganización de las tareas agrícolas, observamos por otro lado la emergencia de una agricultura comercial orientada hacia el mercado urbano regional. Procediendo a la descripción de fotografías tomadas en la campo como en la ciudad, procuraremos explicar el conjunto de estas mutaciones, antes de preguntarnos más ampliamente sobre el futuro de las explotaciones familiares en esta región. 
INDEX

Mots-clés : agriculture familiale, Andes, approvisionnement urbain, Equateur, migration

Palabras claves : agricultura familiar, Andes, aprovisionamiento urbano, Ecuador, migración

\section{AUTEUR}

\section{NASSER REBAÏ}

Nasser Rebaï, nass.reb@hotmail.fr, est ATER à l'Université Paris 1 Panthéon-Sorbonne et membre de l'UMR 8586 PRODIG-CNRS 